\title{
First-in-human implantation of a novel transfemoral self- expanding transcatheter heart valve to treat pure aortic regurgitation
}

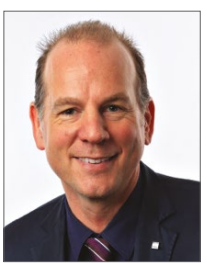

Ulrich Schäfer, MD; Johannes Schirmer, MD; Niklas Schofer, MD; Eva Harmel, MD;

Florian Deuschl, MD; Lenard Conradi, MD

University Heart Center, Hamburg, Hamburg, Germany

\section{KEYWORDS}

- aortic regurgitation

- elderly (>75 years)

- femoral

- transcatheter

aortic valave

implantation (TAVI)

\section{Abstract}

Here we will describe the first case of a novel JenaValve design (JenaValve Technology, Irvine, CA, USA) using the transfemoral approach in a 78-year-old female patient with pure aortic regurgitation. The implantation was successfully performed with good haemodynamics and good clinical outcome at six-month follow-up. The technology is especially appealing in non-calcified anatomies due to the clipping mechanism which was previously utilised in the transapical design.

*Corresponding author: Department of Cardiology, Martinistrasse 52, 20246 Hamburg, Germany. 


\section{Abbreviations}

AR aortic regurgitation

LV left ventricular

NYHA New York Heart Association

TAVI transcatheter aortic valve implantation

\section{Introduction}

Transcatheter aortic valve implantation (TAVI) is an established treatment option for severe aortic stenosis in patients at high or prohibitive surgical risk. In addition, TAVI has also been used to treat pure aortic regurgitation (AR) ${ }^{1,2}$. Several cases or series have been reported using various transcatheter heart valves (THV). Because most THV usually require a certain extent of calcification for anchoring, their use in native primary AR is limited.

In this regard, transapical treatment with the JenaValve (JenaValve Technology, Irvine, CA, USA), a self-expanding porcine root valve with three integrated locators (formerly named "feelers"), has been demonstrated to have particular value in patients with native primary $\mathrm{AR}^{3}$.

Anatomically correct positioning within the native cusps and clipping of the THV onto the native leaflets led to a CE mark being granted to this device for this particular indication in $2013^{3}$. In addition, rapid pacing is not required due to the device design and implant technique. The JenaValve does not need calcification of the native valve for anchoring of the prosthesis and thus seems well suited for use in AR-dominant disease. Its additional value in treating aortic stenosis has also repeatedly been shown in recent publications ${ }^{4,5}$.

In this report, we describe the first case of a successful implantation of a novel JenaValve THV design (Figure 1A) ${ }^{6}$ composed of porcine pericardial leaflets mounted on a self-expanding nitinol stent with three locators, using the transfemoral approach in a patient with pure AR.

\section{Methods and results}

A 78-year-old woman was admitted to our institution presenting with worsening symptoms (NYHA Class III-IV) related to chronic severe native primary AR (Figure 1B). Transthoracic echocardiography revealed a slightly enlarged left ventricle with a left ventricular (LV) end-diastolic diameter of $58 \mathrm{~mm}$, LV end-systolic diameter of $43 \mathrm{~mm}$, and an ejection fraction of $48 \%$. A multislice spiral computed tomographic scan revealed a normally configured aortic root and ascending aorta without any calcification of the aortic valvular cusps (Figure 1C). The coronary angiogram displayed no significant coronary artery disease. Concomitant comorbidities

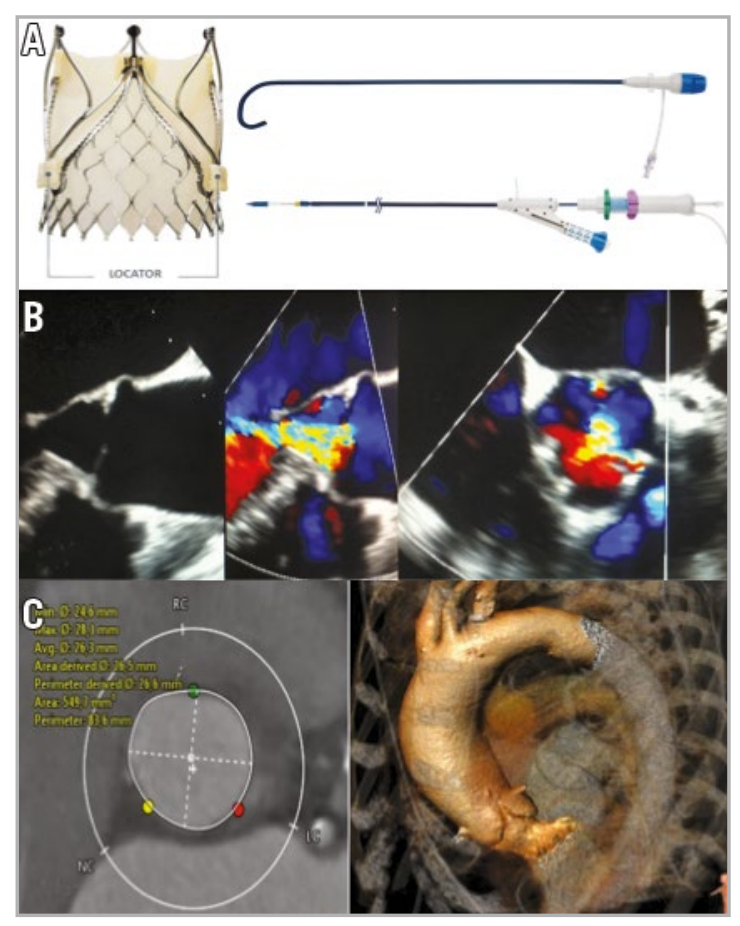

Figure 1. JenaValve technology, transoesophageal echocardiography and multislice computed tomography (MSCT). A) Left. Porcine pericardial tissue valve with a low stent profile that achieves true anatomical alignment with the native commissures, thereby mitigating the risk of coronary obstruction and valve dislocation with the unique locator technology. A) Right top. 19 Fr pre-shaped and hydrophilic coated introducer system with a haemostatic valve and dilator incorporating an atraumatic tip allowing valve delivery into the ascending aorta above the native valve. A) Right bottom. Delivery catheter with an ergonomic handle and integrated deflection catheter. B) Transoesophageal echocardiography colour Doppler images before treatment showing severe central aortic regurgitation. C) Multislice computed tomography showing a round annular enlargement (perimeter $83.6 \mathrm{~mm}$ ) without calcification and a slight enlargement of the ascending aorta $(43 \mathrm{~mm})$. Sizing is solely derived from MSCT with an oversizing usually in the range of between 10 and $20 \%$ (sizing chart). 
were atrial fibrillation, cachexia/frailty, rheumatoid arthritis, pulmonary hypertension (sPAP 55-65 mmHg) and mild renal insufficiency. Despite a relatively low EuroSCORE I of $13.9 \%$ and a Society of Thoracic Surgeons score of $2.5 \%$, the patient was deemed a candidate for a transcatheter procedure due to significant frailty and other comorbidities not captured in the scoring tools.

Having obtained informed consent, a transfemoral valve implantation was performed in a hybrid operating room. Transfemoral access was obtained in the standard manner (pre-closure technique). Because of the known low contrast visibility in conditions of AR, two pigtail catheters were introduced for better visualisation. Valvuloplasty was omitted; the size 27 THV was directly inserted into the annulus with the aid of the specifically designed delivery system. The three locators were exposed and placed at the base of the aortic cusps. Correct positioning was confirmed solely with fluoroscopy (Figure 2A) using different MSCT-derived precalculated planes to visualise all three aortic cusps in relation to the locators (Figure 2B). In a second step, the prosthesis was deployed without pacing. The nitinol stent expanded and was safely anchored in the native valve annulus (Figure 2C). No paravalvular leak was found on angiographic (Figure 2C) and immediate echocardiographic control. The femoral access site was closed in routine fashion. After successful valve implantation (fluoroscopy time $37 \mathrm{~min}, 175 \mathrm{cc}$ contrast medium), the patient demonstrated an immediate haemodynamic improvement and was subsequently transferred to the intensive care unit. Discharge from the hospital was possible on postoperative day 9. Unfortunately, the patient developed mild right-sided hemiparesis on post-procedure day 1 . This observation was probably related to atrial fibrillation with subtherapeutic periprocedural anticoagulation treatment. Fortunately, the patient was discharged home without a significant neurological deficit in a significantly improved functional state (NYHA Class II) without any AR. Repeat echocardiographic studies at 30 days, three months and six months demonstrated a persistent good result with a valve mean gradient of $8-9 \mathrm{mmHg}$ and no paravalvular leak.

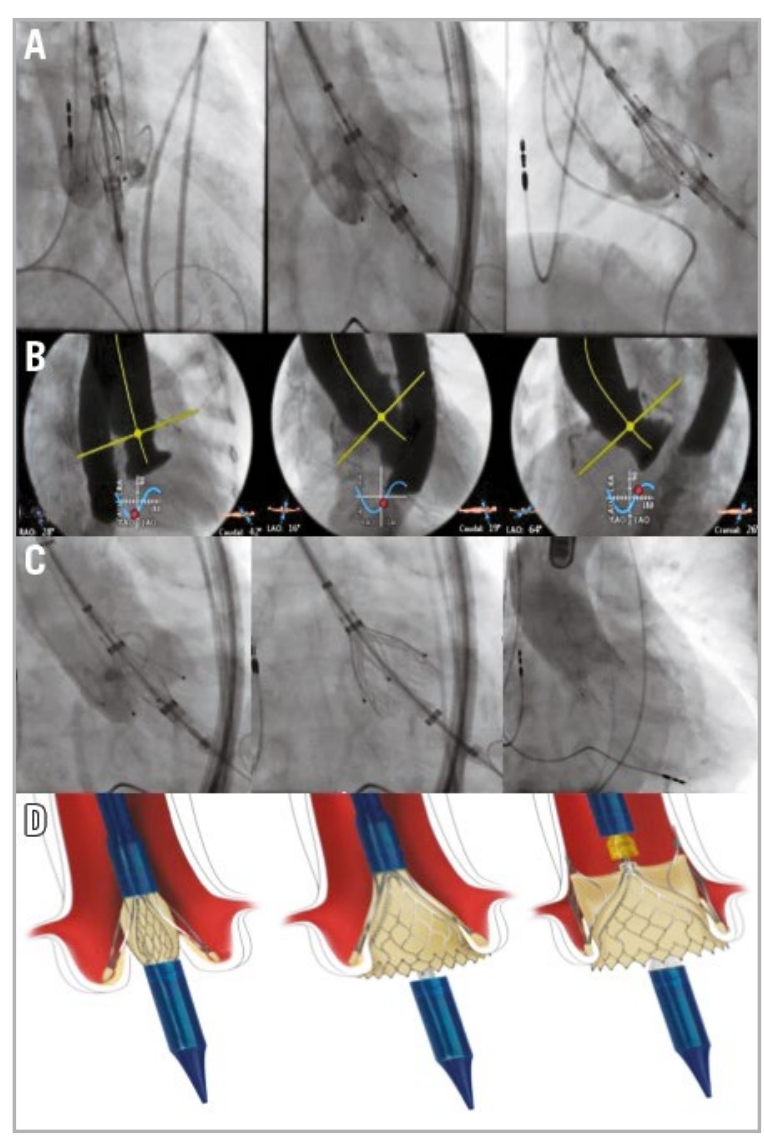

Figure 2. Fluoroscopy and MSCT-derived C-arm angulations, drawings of JenaValve anchoring. A) The size 27 THV was directly inserted into the annulus with the aid of the specifically designed delivery system. The three locators were exposed and placed at the base of the aortic cusps. B) Angulation of the C-arm was pre-calculated by MSCT and positioning of the locators in relation to the cusps was confirmed by fluoroscopy. C) Fluoroscopy during implantation of the JenaValve. Left, the three locators are placed in the native cusps. Middle, the inflow portion is deployed sealing the native leaflets against the stent (paper clip mechanism, oversizing by $10-20 \%$ is sufficient for sealing). Right, final angiography with no valvular or paravalvular aortic regurgitation. D) Schematic drawings demonstrating the unique "paper clip mechanism" using the native leaflets for sealing. 


\section{Discussion}

With next-generation valves such as the JenaValve, a further expansion of the indication for TAVI can be expected. The special implant mechanism of the JenaValve remains currently the most appealing concept for patients without calcification of the aortic cusps. By design, an oversizing of $10-20 \%$ is sufficient for sealing due to the unique "paper clip mechanism" using the native leaflets for sealing (Figure 2D). The presented case demonstrates that transfemoral implantation of a JenaValve pericardial THV is feasible and seems to be similarly promising as a treatment option for patients with primary AR and high surgical operative risk.

\section{Limitations}

This is a single case report with limited information on the overall applicability of this technology.

\section{Conclusions}

Since the transapical JenaValve system was removed from the market solely due to the significant decline of transapical access utilisation, broader clinical experience and longer-term data with regard to the performance of this transfemoral valve design for this indication are needed.

\section{Impact on daily practice}

Unfortunately, this transcatheter heart valve is not available for clinical use outside of clinical studies, but we believe that it has a particular value in patients with primary $A R$.

\section{Conflict of interest statement}

U. Schäfer, J. Schirmer and L. Conradi are proctors for JenaValve Technology and our institution has received grant support from JenaValve Technology. The other authors have no conflicts of interest to declare.

\section{References}

1. Walther T, Thielmann M, Kempfert J, Schroefel H, WimmerGreinecker G, Treede H, Wahlers T, Wendler O. One-year multicentre outcomes of transapical aortic valve implantation using the SAPIEN XTтм valve: the PREVAIL transapical study. Eur $J$ Cardiothorac Surg. 2013:43:986-92.

2. Bleiziffer S, Mazzitelli D, Nöbauer C, Ried T, Lange R. Successful treatment of pure aortic insufficiency with transapical implantation of the JenaValve. Thorac Cardiovasc Surg. 2013;61: 428-30.

3. Seiffert M, Bader R, Kappert U, Rastan A, Krapf S, Bleiziffer S, Hofmann S, Arnold M, Kallenbach K, Conradi L, Schlingloff F, Wilbring M, Schäfer U, Diemert P, Treede H. Initial German experience with transapical implantation of a second-generation transcatheter heart valve for the treatment of aortic regurgitation. JACC Cardiovasc Interv. 2014;7:1168-74.

4. Treede H, Mohr FW, Baldus S, Rastan A, Ensminger S, Arnold M, Kempfert J, Figulla HR. Transapical transcatheter aortic valve implantation using the JenaValve ${ }^{\mathrm{TM}}$ system: acute and 30-day results of the multicentre CE-mark study. Eur J Cardiothorac Surg. 2012;41:e131-8.

5. Sawaya FJ, Deutsch MA, Seiffert M, Yoon SH, Codner P, Wickramarachchi U, Latib A, Petronio AS, Rodés-Cabau J, Taramasso M, Spaziano M, Bosmans J, Biasco L, Mylotte D, Savontaus M, Gheeraert P, Chan J, Jørgensen TH, Sievert H, Mocetti M, Lefèvre T, Maisano F, Mangieri A, Hildick-Smith D, Kornowski R, Makkar R, Bleiziffer S, Søndergaard L, De Backer O. Safety and Efficacy of Transcatheter Aortic Valve Replacement in the Treatment of Pure Aortic Regurgitation in Native Valves and Failing Surgical Bioprostheses: Results From an International Registry Study. JACC Cardiovasc Interv. 2017;10:1048-56.

6. Rudolph TK, Baldus S. JenaValve - transfemoral technology. EuroIntervention. 2013;9 Suppl:S101-2. 